\title{
Collaborating Reviewers
}

Invited reviewers are asked to identify colleagues who assisted with review. eNeuro would like to acknowledge the hard work of these collaborating reviewers and thank them for their service to the journal.

Mohammad Abdolrahmani
Andrew Alegria
Navonil Banerjee
Sebastiano Bariselli
Lily Barrett
Laura Benoit
Kaitlin Best
Sonia Betti
Anthony Bilodeau
Caroline Biojone
Elizabeth Brija
Kathleen Bryant
Zach Chia
Zahraa Chorghay
Wei Dai
Javier Zorrilla de San Martin
Alessandra Donato
Marion Ducret
Yonatan Fatal
Maria Agustina Frechou
Jude Frie
Michael Gongwer
Steven Granger
Pedro Guedes
Umair Hassan

Maximillan Hauser
Jessica Higgibotham
Xue Yan Ho
Victoria Hones
Andrew Gregory Howe
Luyang Hua
Eric J. R. Jansen
Elizabeth Johnson
Kevin Johnston
Amey Joshi
Maxim Katsenelson
Ashley Kees
Clemens Kiecker
Eena Kosik
Yi-Chen Lai
Renzo Lanfanco
Hudson Lin
Yu Liu
Annie Ly
Sara Mahallati
Erin Maher
David Martin
Sakthikumar Mathivanan
Michelle McCarthy
Alex McCoy

$\begin{array}{ll}\text { Kelsey McDermott } & \text { Tomoya Shibahara } \\ \text { Dillon McGovern } & \text { Marissa Smail } \\ \text { Swarup Mitra } & \text { Rebecca Somach } \\ \text { Aleisha Moore } & \text { Elliot Sprecher } \\ \text { Pablo Munoz } & \text { Joan Stilling } \\ \text { Stefano Musardo } & \text { Jasmin Strickland } \\ \text { Kuniyuki Nakamura } & \text { Yosuke Tanaka } \\ \text { Jacques Nguyen } & \text { Can Tao } \\ \text { Suzanne Nolan } & \text { Jelena Trajkovic } \\ \text { Mitchell Nothem } & \text { Troy Trevino } \\ \text { Alvaro Nuno } & \text { Alexandra Tsolias } \\ \text { Kenichi Okada } & \text { Catherine Ubri } \\ \text { Mattia Pagnotta } & \text { Juzoh Umemori } \\ \text { Francois Pauzin } & \text { Milan Valyear } \\ \text { Caterina Pedersini } & \text { Jonathan VanRyzin } \\ \text { Dina Popova } & \text { James Walton } \\ \text { Yi Valerie Pu } & \text { Danying Wang } \\ \text { Zhen Qui } & \text { Frederike Winkel } \\ \text { Ravjot Rehsi } & \text { Tatiana Wolfe } \\ \text { Catalina Requejo } & \text { Xiaxia Xu } \\ \text { Lace Riggs } & \text { Alex Yu } \\ \text { Chad Sauvola } & \text { Idit Yvgi } \\ \text { Raffael Schmitt } & \text { Shenyu Zhai } \\ \text { Lauren Seabrook } & \text { Zihao Zhang } \\ \text { Weixing Shen } & \text { Judy Zhu } \\ & \\ & \end{array}$

